\title{
Functions of bounded variation and the AM-modulus in R-n
}

\section{Exnerova, Vendula Honzlova}

2018-12

Exnerova , V H , Maly , J \& Martio , O 2018, ' Functions of bounded variation and the AM-modulus in R-n ' , Nonlinear Analysis: Theory, Methods \& Applications , vol. 177 , pp. 553-571 . https://doi.org/10.1016/j.na.2018.05.015

http://hdl.handle.net/10138/322149

https://doi.org/10.1016/j.na.2018.05.015

cc_by_nc_nd

acceptedVersion

Downloaded from Helda, University of Helsinki institutional repository.

This is an electronic reprint of the original article.

This reprint may differ from the original in pagination and typographic detail.

Please cite the original version. 


\title{
FUNCTIONS OF BOUNDED VARIATION AND THE $A M-$ MODULUS IN $\mathbb{R}^{n}$
}

\author{
VENDULA HONZLOVÁ EXNEROVÁ, JAN MALÝ, AND OLLI MARTIO
}

\begin{abstract}
Moduli of path families are widely used to study Sobolev functions. Similarly, the recently introduced approximation $(A M-)$ modulus is helpful in the theory of functions of bounded variation $(B V)$ in $\mathbb{R}^{n}$ [14]. We continue this direction of research. Let $\Gamma_{E}$ be the family of all paths which meet $E \subset \mathbb{R}^{n}$. We introduce the outer measure $E \mapsto A M\left(\Gamma_{E}\right)$ and compare it with other $(n-1)$-dimensional measures. In particular, we show that $A M\left(\Gamma_{E}\right)=2 \mathcal{H}^{n-1}(E)$ whenever $E$ lies on a countably $(n-1)-$ rectifiable set. Further, we study functions which have bounded variation on $A M$-a.e. path and we relate these functions to the classical $B V$ functions which have only bounded essential variation on $A M$-a.e. path. We also characterize sets $E$ of finite perimeter in terms of the $A M$-modulus of two path families naturally associated with $E$.
\end{abstract}

\section{INTRODUCTION}

The approximation modulus, abbreviated as $A M$-modulus, was introduced in [14] to study functions of bounded variation $(B V)$ in metric measure spaces, see also [11] and [15]. The $A M$-modulus offers a counterpart to the Fuglede theorem [10] which states that functions in the Sobolev space $W^{1, p}\left(\mathbb{R}^{n}\right), p \geq 1$, are absolutely continuous on every path in $\mathbb{R}^{n}$ except of a family whose $M_{p}$-modulus is zero and in [14] it was shown that $B V$ functions in metric measure spaces have bounded essential variation on $A M$ almost every path. In Section 4 we show that $B V$ functions in $\mathbb{R}^{n}$ have bounded variation in the classical sense, and not only bounded essential variation, on $A M$ almost every path in $\mathbb{R}^{n}$. This leads to a new characterization of $B V$ functions in $\mathbb{R}^{n}$.

Let $\Gamma_{E}$ denote the family of all paths which meet a set $E \subset \mathbb{R}^{n}$. It turns out that the set function $E \mapsto A M\left(\Gamma_{E}\right)$ is a non-trivial measure in $\mathbb{R}^{n}$ and we compare this to the $(n-1)$-dimensional Hausdorff measure $\mathcal{H}^{n-1}(E)$ and pay special attention to the case when $E$ lies on a "regular" set $A \subset \mathbb{R}^{n}$, for instance on a countably $(n-1)$-rectifiable set $A$ in Section 5.

In the last section, Section 6, we give two characterizations for sets $E$ of finite perimeter in terms of the $A M$-modulus. The perimeter of $E$

VHE and JM have been supported by the grant GA ČR P201/18-07996S of the Czech Science Foundation. 
coincides with the $A M$-modulus of the family of curves connecting the measure theoretic interior to the measure theoretic exterior of $E$ and with the $A M$-modulus of the family of curves $\gamma:[a, b] \rightarrow \mathbb{R}^{n}$ which meet the measure theoretic boundary of $E$ at some point $\gamma(t), t \in(a, b)$.

Note that there is a parallel approach to measuring curves [4], which is related to modulus [2] and can be also used to characterize $B V$ spaces [1].

Our notation is standard and more specialized symbols and concepts are explained in due course.

\section{Preliminaries and $A M-$ Modulus}

Let $A \subset \mathbb{R}$ and $f: A \rightarrow[-\infty, \infty]$. The total variation of $f$ on $A$ is

$$
\begin{aligned}
& V(f, A)=\sup \left\{\sum_{j=1}^{m}\left|f\left(x_{j}\right)-f\left(x_{j-1}\right)\right|:\right. \\
& \left.x_{0}<x_{1}<x_{2}<\ldots<x_{m}, \quad x_{j} \in A\right\}
\end{aligned}
$$

where $\left|f\left(x_{j}\right)-f\left(x_{j-1}\right)\right|=\infty$ if $\left|f\left(x_{j}\right)-f\left(x_{j-1}\right)\right|$ is undefined, i.e. of the form $|\infty-\infty|$. If $A$ is (Lebesgue) measurable and $f$ is measurable in $A$, then the essential variation of $f$ on $A$ is

$$
\tilde{V}(f, A)=\inf \{V(u, A): u=f \text { a.e. in } A\} ;
$$

obviously $\tilde{V}(f, A) \leq V(f, A)$ and $\tilde{V}(f,[a, b])=\tilde{V}(f,(a, b))$. If $f \in$ $L_{\text {loc }}^{1}((a, b))$, then by $[3$, Theorem 3.27], see also [8, 5.10.1 Theorem 1],

$$
\tilde{V}(f,(a, b))=\sup \left\{\int_{A} f \varphi^{\prime} d t: \varphi \in C_{0}^{1}((a, b)),|\varphi| \leq 1\right\} .
$$

For the properties of $V(f, A)$ and $\tilde{V}(f, A)$ see e.g. [3, Section 3.2], [8, Section 5.10.1] and [14].

A continuous mapping $\gamma:[a, b] \rightarrow \mathbb{R}^{n}$ is called a curve. We say that a curve $\gamma$ is a path if it has a finite and non-zero total length; in this case we parametrize $\gamma$ by its arclength. We consider paths with various lengths but (with some abuse of notation) do not mark the dependence of the total length $\ell$ on $\gamma$. The locus of $\gamma$ is defined as $\gamma([0, \ell])$ and denoted by $\langle\gamma\rangle$.

If $\gamma$ is a path and $f:\langle\gamma\rangle \rightarrow[-\infty, \infty]$, we write $V(f, \gamma)=V(f \circ$ $\gamma,[0, \ell])$ and $\tilde{V}(f, \gamma)=\tilde{V}(f \circ \gamma,[0, \ell])$ provided that $f \circ \gamma$ is measurable on $[0, \ell]$.

We refer to [14] and [11] for the properties of the $A M$-modulus and to [5] and [10] for those of the $M_{p}$-modulus. For completeness we recall the definition for the $A M$-modulus. Let $\Gamma$ be a family of paths in $\mathbb{R}^{n}$. A sequence $\left(\rho_{i}\right)$ of Borel functions on $\mathbb{R}^{n}$ with values in $[0, \infty]$ is said 
to be $A M$-admissible (or simply admissible) for $\Gamma$ if for every $\gamma \in \Gamma$

$$
\liminf _{i} \int_{\gamma} \rho_{i} d s \geq 1
$$

and we define the $A M$-modulus of $\Gamma$ as

$$
A M(\Gamma):=\inf \left\{\liminf _{i} \int_{\mathbb{R}^{n}} \rho_{i} d x:\left(\rho_{i}\right)_{i} \text { is admissible for } \Gamma\right\} .
$$

The $A M$-modulus, as the $M_{p}$-modulus, is monotone and countably subadditive and we say that a property holds for $A M$-a.e. curve if the property fails on the family $\Gamma$ such that $A M(\Gamma)=0$.

With an arbitrary set $E \subset \mathbb{R}^{n}$ we associate the family $\Gamma_{E}$ of all paths that meet $E$. Further, let $\Gamma_{E}^{c}$ be the family of all paths $\gamma$ in $\mathbb{R}^{n}$ that cross $E$, i.e. there is an interior point $t \in(0, \ell)$ such that $\gamma(t) \in E$. Although $\Gamma_{E}$ looks more natural, in the subsequent sections it becomes evident that $A M\left(\Gamma_{E}^{c}\right)$ is better related to the $(n-1)$-dimensional Hausdorff measure in $\mathbb{R}^{n}$ than $A M\left(\Gamma_{E}\right)$.

The following lemma clarifies the roles of $\Gamma_{E}$ and $\Gamma_{E}^{c}$.

Lemma 1. $A M\left(\Gamma_{E}\right)=2 A M\left(\Gamma_{E}^{c}\right)$.

Proof. Let $\left(\rho_{i}\right)$ be an $A M$-admissible sequence for $\Gamma_{E}$ and $\gamma \in \Gamma_{E}^{c}$. Since $\gamma\left(t_{0}\right) \in E$ for some $\left.t_{0} \in(0, \ell)\right)$, the paths $\gamma_{1}=\gamma L_{\left[0, t_{0}\right]}$ and $\gamma_{2} L_{\left[t_{0}, \ell\right]}$ belong to $\Gamma_{E}$ and hence

$$
\begin{gathered}
\liminf _{i} \int_{\gamma} \rho_{i} d s=\liminf _{i}\left(\int_{\gamma_{1}} \rho_{i} d s+\int_{\gamma_{2}} \rho_{i} d s\right) \\
\geq \liminf _{i} \int_{\gamma_{1}} \rho_{i} d s+\liminf _{i} \int_{\gamma_{2}} \rho_{i} d s \geq 2 .
\end{gathered}
$$

Thus the sequence $\left(\rho_{i} / 2\right)$ is $A M$-admissible for $\Gamma_{E}^{c}$ and we obtain $A M\left(\Gamma_{E}^{c}\right) \leq A M\left(\Gamma_{E}\right) / 2$.

For the reverse inequality let $\left(\rho_{i}^{c}\right)$ be an $A M$-admissible sequence for $\Gamma_{E}^{c}$ and let $\gamma \in \Gamma_{E} \backslash \Gamma_{E}^{c}$. We can assume that $\gamma(\ell) \in E$. Let $\tilde{\gamma}$ be the path $\tilde{\gamma}(t)=\gamma(t)$ for $t \in[0, \ell]$ and $\tilde{\gamma}(t)=\gamma(2 \ell-t)$ for $t \in(\ell, 2 \ell]$. Then $\tilde{\gamma} \in \Gamma_{E}^{c}$ and

$$
1 \leq \liminf _{i} \int_{\tilde{\gamma}} \rho_{i}^{c} d s=2 \liminf _{i} \int_{\gamma} \rho_{i}^{c} d s .
$$

Thus $\left(2 \rho_{i}^{c}\right)$ is an $A M$-admissible sequence for $\Gamma_{E}$ and, consequently, $A M\left(\Gamma_{E}\right) \leq 2 A M\left(\Gamma_{E}^{c}\right]$.

The following auxiliary lemma is often useful.

Lemma 2. If $U \subset \mathbb{R}^{n}$ is open and $E \subset U$, then $A M\left(\Gamma_{E}\right)=A M\left(\Gamma_{E}(U)\right)$ where $\Gamma_{E}(U)=\left\{\gamma \in \Gamma_{E}:\langle\gamma\rangle \subset U\right\}$.

Proof. If $\gamma \in \Gamma_{E} \backslash \Gamma_{E}(U)$, then $\gamma$ has a subpath $\tilde{\gamma} \in \Gamma_{E}(U)$ and thus $A M\left(\Gamma_{E}(U)\right) \geq A M\left(\Gamma_{E}\right)$. On the other hand, $\Gamma_{E}(U) \subset \Gamma_{E}$ and thus $A M\left(\Gamma_{E}(U)\right) \leq A M\left(\Gamma_{E}\right)$. 
For $E \subset \mathbb{R}^{n}$ we set $\phi(E)=A M\left(\Gamma_{E}^{c}\right)=\frac{1}{2} A M\left(\Gamma_{E}\right)$ and call $\phi$ the $A M$-modulus measure. The following theorem justifies the name.

Theorem 1. $\phi$ is a metric outer measure on $\mathbb{R}^{n}$. Therefore, all Borel sets are $\phi$-measurable.

Proof. Obviously $\phi(\emptyset)=0$ and $\phi$ is monotone. For the countable subadditivity let $E=\cup_{j} E_{j}$. We may assume that $\phi(E)<\infty$. Choose $\varepsilon>0$. For each $j$ find an $A M$-admissible sequence $\left(\rho_{i}^{j}\right)_{i}$ for $\Gamma_{E_{j}}^{c}$ such that

$$
\int_{\mathbb{R}^{n}} \rho_{i}^{j} d \mu \leq A M\left(\Gamma_{E_{j}}^{c}\right)+2^{-j} \varepsilon
$$

for each $j$ and $i$. This is possible by passing to subsequences. Now

$$
\rho_{i}=\sum_{j} \rho_{i}^{j}
$$

is $A M$-admissible for $\Gamma_{E}^{c}$ and we obtain

$$
\begin{gathered}
\phi(E)=A M\left(\Gamma_{E}^{c}\right) \leq \liminf _{i} \int_{\mathbb{R}^{n}} \rho_{i} d x \\
\leq \liminf _{i}\left(\sum_{j} A M\left(\Gamma_{E_{j}}^{c}\right)+2^{-j} \varepsilon\right) \leq \sum_{j} A M\left(\Gamma_{E_{j}}^{c}\right)+\varepsilon
\end{gathered}
$$

and letting $\varepsilon \rightarrow 0$ we obtain

$$
\phi(E)=A M\left(\Gamma_{E}^{c}\right) \leq \sum_{j} \phi\left(E_{j}\right)
$$

as required. We have shown that $\phi$ is an outer measure in $\mathbb{R}^{n}$.

It remains to show that $\phi$ is a metric outer measure, i.e. if sets $E_{1}, E_{2} \subset \mathbb{R}^{n}$ are such that $d\left(E_{1}, E_{2}\right)>\delta$ for some $\delta>0$, then

$$
\phi\left(E_{1}\right)+\phi\left(E_{2}\right)=\phi\left(E_{1} \cup E_{2}\right) .
$$

Now there are disjoint open sets $U_{i}, i=1,2$, with $E_{i} \subset U_{i}$ and then (2) follows from Lemma 2. Therefore $\phi$ is a metric outer measure and, consequently, all Borel sets are $\phi$-measurable.

For the set theoretic terminology below we refer to $[9,2.2 .10]$ and [13].

Theorem 2. Let $E \subset \mathbb{R}^{n}$ be an arbitrary set. Then there exists a $\phi$ measurable (co-Suslin) set $F \subset \mathbb{R}^{n}$ such that $E \subset F$ and $\phi(F)=\phi(E)$. The AM-modulus measure $\phi$ also has this property.

Proof. In this proof we use the version $\phi(E)=\frac{1}{2} A M\left(\Gamma_{E}\right)$ of the definition of $\phi$. Since closed sets are $\phi$-measurable, each co-Suslin set is $\phi$ measurable, [9, Theorem 2.2.12]. We follow the proof of Proposition 1.5 in [6]. Let $\mathcal{L}$ be the space of all curves $\gamma:[0,1] \rightarrow \mathbb{R}^{n}$ with Lipschitz constant $\leq 1$ equipped with the distance $d\left(\gamma_{1}, \gamma_{2}\right)=\sup _{t \in[0,1]}\left|\gamma_{1}(t)-\gamma_{2}(t)\right|$. 
Then $\mathcal{L}$ is a complete metric space. If $\left(\rho_{i}\right)$ is an admissible sequence for $\Gamma_{E}$, consider the mapping $\Phi_{i}: \mathcal{L} \rightarrow[0, \infty]$,

$$
\Phi_{i}(\gamma)=\int_{\gamma} \rho_{i} d s, \quad \gamma \in \mathcal{L}
$$

Then $\Phi_{i}$ are Borel measurable, see [12] (see also [6]), and thus

$$
\mathcal{L}_{1}=\left\{\gamma \in \mathcal{L}: \gamma \text { is nonconstant, } \liminf _{i} \Phi_{i}(\gamma)<1\right\}
$$

is also a Borel set in $\mathcal{L}$. Now, the evaluation mapping $\kappa: \gamma \mapsto \gamma(0)$ is continuous and thus $G=\kappa\left(\mathcal{L}_{1}\right)$ is a Suslin set. Set $F=\mathbb{R}^{n} \backslash G$. If $x \in G \cap E$, then there exists $\gamma \in \mathcal{L}_{1}$ such that $\gamma(0)=x$. Thus $\gamma \in \Gamma_{E}$, which is a contradiction, as $\mathcal{L}_{1} \cap \Gamma_{E}=\emptyset$. Hence $E \subset F$. The sequence $\left(\rho_{i}\right)$ is admissible for $\Gamma_{F}$ and thus

$$
\phi(F) \leq \frac{1}{2} \liminf _{i} \int_{\mathbb{R}^{n}} \rho_{i} d x .
$$

Taking infimum over all admissible sequences we obtain $\phi(F) \leq \phi(E)$, whereas the converse inequality is obvious because $E \subset F$.

Remark 1. Lemmata 1 and 2 and Theorems 1 and 2 hold in metric measure spaces with similar proofs.

We need estimates between $\phi(E)=A M\left(\Gamma_{E}^{c}\right)$ and the $(n-1)-$ dimensional Hausdorff measure

$$
\mathcal{H}^{n-1}(E)=\sup _{\delta>0} \mathcal{H}_{\delta}^{n-1}(E)
$$

where

$\mathcal{H}_{\delta}^{n-1}(E)=\inf \left\{2^{1-n} \boldsymbol{\alpha}_{n-1} \sum_{i=1}^{\infty}\left(\operatorname{diam} E_{i}\right)^{n-1}: E \subset \bigcup_{i=1}^{\infty} E_{i}, \operatorname{diam} E_{i}<\delta\right\}$ are the Hausdorff $\delta$-contents and $\boldsymbol{\alpha}_{n-1}=\mathcal{L}^{n-1}\left(B^{n-1}(0,1)\right)$.

Let $G(n, n-1)$ be the Grassmannian manifold of $(n-1)$-dimensional linear subspaces of $\mathbb{R}^{n}$. With each $V \in G(n, n-1)$ we associate the orthogonal projection $\Pi_{V}: \mathbb{R}^{n} \rightarrow V$. For $E \subset \mathbb{R}^{n}$ we define

$$
\mathcal{G}^{n-1}(E)=\sup _{\delta>0} \mathcal{G}_{\delta}^{n-1}(E)
$$

where

$$
\begin{aligned}
\mathcal{G}_{\delta}^{n-1}(E)= & \inf \left\{\sum_{i=1}^{\infty} \sup \left\{\mathcal{H}^{n-1}\left(\Pi_{V}\left(E_{i}\right)\right): V \in G(n, n-1)\right\}:\right. \\
& \left.E_{i} \text { Borel, } E \subset \bigcup_{i=1}^{\infty} E_{i}, \operatorname{diam} E_{i}<\delta\right\} .
\end{aligned}
$$

This is the $(n-1)$-dimensional Gross measure, see [9, 2.10.4].

We let $\mathbb{H}_{n}$ denote the coordinate plane $\left\{x \in \mathbb{R}^{n}: x_{n}=0\right\}$ and identify $\mathbb{R}^{n-1}=\mathbb{H}_{n}$. 
Inequality (3) below (with a different constant) was considered in metric measure spaces in [14, Theorem 3.17].

Theorem 3. If $E \subset \mathbb{R}^{n}, n>1$, then

$$
\phi(E) \leq c_{n} \mathcal{H}^{n-1}(E),
$$

where

$$
c_{n}=\frac{n^{n} \boldsymbol{\alpha}_{n}}{2(n-1)^{n-1} \boldsymbol{\alpha}_{n-1}} .
$$

Proof. We may assume that $\mathcal{H}^{n-1}(E)<\infty$. For $j=1,2$, ... choose a covering $E_{i}^{j}, i=1,2, \ldots$, of $E$ such that $d_{i}^{j}:=\operatorname{diam} E_{i}^{j} \leq 2^{-j}$ and

$$
2^{1-n} \boldsymbol{\alpha}_{n-1} \sum_{i=1}^{\infty}\left(d_{i}^{j}\right)^{n-1} \leq \mathcal{H}_{2^{-j}}^{n-1}(E)+2^{-j} \leq \mathcal{H}^{n-1}(E)+2^{-j}
$$

Set

$$
\rho_{j}(x)=\sum_{i} \frac{1}{2 r_{i}^{j}} \chi_{E_{i}^{j}+B_{i}^{j}}(x)
$$

where $B_{i}^{j}=B\left(0, r_{i}^{j}\right)$ and

$$
r_{i}^{j}=\frac{1}{2(n-1)} d_{i}^{j}
$$

Then $\rho_{j}$ are Borel functions and the sequence $\left(\rho_{j}\right)$ is $A M$-admissible for $\Gamma_{E}^{c}$. Indeed, if $\gamma \in \Gamma_{E}^{c}$, then there is $t_{0} \in(0, \ell)$ such that $\gamma\left(t_{0}\right) \in E$. The path $\gamma L_{\left[0, t_{0}\right]}$ is non-constant and hence there is $j_{0}$ such that for $j \geq j_{0}, \gamma\left\lfloor_{\left[0, t_{0}\right]}\right.$ meets both $E_{i}^{j}$ and the complement of $E_{i}^{j}+B_{i}^{j}$ for some $i=i(j)$. Now the path $\gamma L_{\left[0, t_{0}\right]}$ travels in $E_{i}^{j}+B_{i}^{j}$ at least distance $r_{i}^{j}$. The same applies to the path $\gamma\left\lfloor_{\left[t_{0}, \ell\right]}\right.$, possibly with a different $j_{0}$, and hence $\gamma$ travels in $E_{i}^{j}+B_{i}^{j}$ at least distance $2 r_{i}^{j}$. Thus

$$
\liminf _{j} \int_{\gamma} \rho_{j} d s \geq 1
$$

as required. Using the isodiametric inequality we estimate

$$
\begin{aligned}
A M\left(\Gamma_{E}^{c}\right) & \leq \liminf _{j} \int_{\mathbb{R}^{n}} \rho_{j} d x \leq 2^{-n} \boldsymbol{\alpha}_{n} \liminf _{j} \sum_{i} \frac{1}{2 r_{i}^{j}} \operatorname{diam}\left(E_{i}^{j}+B_{i}^{j}\right)^{n} \\
& =2^{-n} \boldsymbol{\alpha}_{n} \liminf _{j} \sum_{i} \frac{1}{2 r_{i}^{j}}\left(d_{i}^{j}+2 r_{i}^{j}\right)^{n} . \\
& =2^{-n}\left(\frac{n}{n-1}\right)^{n} \boldsymbol{\alpha}_{n} \liminf _{j} \sum_{i} \frac{n-1}{d_{i}^{j}}\left(d_{i}^{j}\right)^{n} \\
& =\frac{n^{n} \boldsymbol{\alpha}_{n}}{2(n-1)^{n-1} \boldsymbol{\alpha}_{n-1}} \liminf _{j} 2^{1-n} \boldsymbol{\alpha}_{n-1} \sum_{i}\left(d_{i}^{j}\right)^{n-1} \\
& \leq \frac{n^{n} \boldsymbol{\alpha}_{n}}{2(n-1)^{n-1} \boldsymbol{\alpha}_{n-1}} \liminf _{j}\left(\mathcal{H}^{n-1}(E)+2^{-j}\right)
\end{aligned}
$$

and (3) follows. 
Theorem 4. For every $V \in G(n, n-1)$ and $E \subset \mathbb{R}^{n}$

$$
\mathcal{H}^{n-1}\left(\Pi_{V}(E)\right) \leq \phi(E)
$$

and if $E$ is a Borel set, then

$$
\mathcal{G}^{n-1}(E) \leq \phi(E) .
$$

Proof. We first prove (4). Let $V \in G(n, n-1)$. We may assume that $V=\mathbb{H}_{n}=\mathbb{R}^{n-1}$. Let $\left(\rho_{i}\right)$ be an $A M$-admissible sequence for $\Gamma_{E}^{c}$. Since each $\rho_{i}$ is a Borel function, the set

$$
A=\left\{y \in \mathbb{R}^{n-1}: \liminf _{i} \int_{-\infty}^{\infty} \rho_{i}(y, t) d t \geq 1\right\}
$$

is a measurable set in $\mathbb{R}^{n-1}$. For each $x^{\prime} \in \Pi_{V}(E)$ choose $x \in \Pi_{V}^{-1}\left(x^{\prime}\right) \cap$ $E$ and define

$$
\gamma_{x^{\prime}}(t)=x+(t-1) e_{n}, \quad t \in[0,2] .
$$

Now $\gamma_{x^{\prime}}$ belongs to $\Gamma_{E}^{c}$. By the Fubini theorem and the Fatou lemma

$$
\begin{gathered}
\liminf _{i} \int_{\mathbb{R}^{n}} \rho_{i} d x \geq \liminf _{i} \int_{A}\left(\int_{-\infty}^{\infty} \rho_{i}((y, t)) d t\right) d y \\
\geq \int_{A}\left(\liminf _{i} \int_{-\infty}^{\infty} \rho_{i}((y, t)) d t\right) d y \geq \mathcal{H}^{n-1}(A) \geq \mathcal{H}^{n-1}\left(\Pi_{V}(E)\right)
\end{gathered}
$$

and since this holds for every $A M$-admissible sequence, the estimate (4) follows.

If $E$ is a Borel set and $\delta>0$, we write $E$ as a pairwise disjoint union of Borel sets $E_{i}$ with diam $E_{i}<\delta$. Using countable subadditivity of $\mathcal{G}_{\delta}^{n-1}$, countable additivity of $\phi$ on Borel sets and (4), we obtain

$$
\mathcal{G}_{\delta}^{n-1}(E) \leq \sum_{i} \mathcal{G}_{\delta}^{n-1}\left(E_{i}\right) \leq \sum_{i} \phi\left(E_{i}\right) \leq \phi(E) .
$$

Passing to the supremum over $\delta$ we obtain (5).

Remark 2. It is easy to see that $\mathcal{H}^{n-1}=\phi$ for $n=1$.

Remark 3. Note that for the estimate (4) we do not need to suppose that $E$ is a Borel set. We do not know if (5) holds for all sets $E \subset \mathbb{R}^{n}$.

\section{3. $B V C$ FUNCTIONS IN $\mathbb{R}^{n}$}

Let $\Omega$ be an open set in $\mathbb{R}^{n}$ and $u: \Omega \rightarrow[-\infty, \infty]$. We say that $u$ is a $B V C$ function, $u \in \mathcal{B V} \mathcal{C}(\Omega)$, if there exists a sequence $\left(g_{i}\right)$ of non-negative Borel functions in $\Omega$ such that

$$
\mid u\left(\gamma(\ell)-u(\gamma(0)) \mid \leq \liminf _{i} \int_{\gamma} g_{i} d s\right.
$$

for each path $\gamma$ in $\Omega$ and

$$
\liminf _{i} \int_{\Omega} g_{i} d x<\infty .
$$


Equation (6) is understood in the sense that if the left hand side is undefined, i.e. of the form $|\infty-\infty|$, then its value is $\infty$. The sequence $\left(g_{i}\right)$ is called a $B V$ upper bound for $u$ in $\Omega$.

Note that we assume no a priori regularity, besides (6), for $u \in$ $\mathcal{B} \mathcal{V C}(\Omega)$. However, in Theorem 5 below we show that $u \in L_{\text {loc }}^{1}(\Omega)$.

If $u \in \mathcal{B V} \mathcal{C}(\Omega)$, then $[14$, Theorem 4.1] shows that for $A M$-a.e. path in $\Omega$ and for every $B V$ upper bound $\left(g_{i}\right)$

$$
V(u, \gamma) \leq \liminf _{i} \int_{\gamma} g_{i} d s<\infty .
$$

The condition (6) can be relaxed and we say that a sequence $\left(g_{i}\right)$ of non-negative Borel functions in $\Omega$ is a weak $B V$ upper bound for $u$ in $\Omega$ if (7) is satisfied and (6) holds for $A M$-a.e. path in $\Omega$. The next lemma shows that weak upper bounds can be used instead of ordinary $B V$ upper bounds.

Lemma 3. If $\left(g_{i}\right)$ is a weak $B V$ upper bound for $u$ and $\varepsilon>0$, then there exists a $B V$ upper bound $\left(\tilde{g}_{i}\right)$ for $u$ and

$$
\liminf _{i} \int_{\Omega} \tilde{g}_{i} d x \leq \liminf _{i} \int_{\Omega} g_{i} d x+\varepsilon .
$$

Proof. Let $\Gamma_{0}$ be the family of all paths in $\Omega$ such that

$$
V(u, \gamma)>\liminf _{i} \int_{\gamma} g_{i} d s .
$$

Since $A M\left(\Gamma_{0}\right)=0$, for each $\varepsilon>0$ there exists a sequence $\left(v_{i}\right)$ of non-negative Borel functions such that

$$
\liminf _{i} \int_{\gamma} v_{i} d s=\infty
$$

for every $\gamma \in \Gamma_{0}$ and

$$
\int_{\Omega} v_{i} d x<\varepsilon
$$

for every $i$, see $\left[11\right.$, Theorem 7 and Remark 6]. Let $\tilde{g}_{i}=g_{i}+v_{i}$. Now $\left(\tilde{g}_{i}\right)$ satisfies 6 and

$$
\liminf _{i} \int_{\Omega} \tilde{g}_{i} d x=\liminf _{i} \int_{\Omega}\left(g_{i}+v_{i}\right) d x \leq \liminf _{i} \int_{\Omega} g_{i} d x+\varepsilon
$$

and so $\left(\tilde{g}_{i}\right)$ is the required $B V$ upper bound for $\mathrm{u}$.

Lemma 3 shows that (8) holds for each weak upper bound for $u \in$ $\mathcal{B} \mathcal{V C}(\Omega)$ and consequently $u$ needs to be defined only in $\Omega \backslash E$ where $\mathrm{E}$ satisfies $A M\left(\Gamma_{E}\right)=0$ and so the values of $u$ on $E$ are immaterial. For example, precisely represented functions $u$ in the Sobolev class $W^{1, p}(\Omega)$, $p \geq 1$, are $B V C$ functions since they are absolutely continuous on $M_{p^{-}}$ a.e. path and hence on $A M$-a.e. path in $\Omega$. The constant sequence $(|\nabla u|)$ is a weak $B V$ upper bound for $u$. 
For an open set $U \subset \Omega$ we define

$$
\begin{aligned}
\left|D_{B V C} u\right|(U)= & \inf \left\{\liminf _{j} \int_{\Omega} g_{i} d x:\right. \\
& \left.\left(g_{i}\right) \text { is a } B V \text { upper bound for } u \text { in } U\right\} .
\end{aligned}
$$

By Lemma 3 weak $B V$ upper bounds can be used as well.

Next we show that a $B V C$ function $u$ in $\Omega$ belongs to $L_{\text {loc }}^{1}(\Omega)$. Since a $B V C$ function has bounded variation on a.e. line parallel to coordinate axis one might think that this property already implies measurability. However, W. Sierpiński [17] constructed a non-measurable set $A \subset \mathbb{R}^{2}$ such every line (not only parallel to coordinate axis) meets $A$ at most in two points. The function $\chi_{A}$ has thus bounded variation on every line segment but it is not measurable. Hence the role of the weak $B V$ upper bound $\left(g_{j}\right)$ is essential.

We employ a weak type estimate for a maximal function associated with a weak $B V$ upper bound $\left(g_{j}\right)$ of a $B V C$ function $u$ in $\Omega$. We set $g_{j}=0$ in $\mathbb{R}^{n} \backslash \Omega$. To simplify the notation, we use the symbol $\mathbf{g}$ for the sequence $\left(g_{j}\right)$ and introduce the maximal function

$$
M \mathbf{g}(x)=\liminf _{j} M g_{j}(x)
$$

where $M g_{j}$ denotes the ordinary Hardy-Littlewood maximal function of $g_{j}$.

Lemma 4. Let $\mathbf{g}=\left(g_{j}\right)$ be a sequence of nonnegative measurable functions on $\mathbb{R}^{n}$ and $t>0$. Then

$$
\mathcal{L}^{n}\left(\left\{x \in \mathbb{R}^{n}: M \mathbf{g}(x)>t\right\}\right) \leq \frac{C_{n}}{t} \liminf _{j} \int_{\mathbb{R}^{n}} g_{j}(z) d z,
$$

where $C_{n}$ depends only on $n$. Consequently, $M \mathbf{g}(x)<\infty$ for a.e. $x \in$ $\mathbb{R}^{n}$.

Proof. Set

$$
I=\liminf _{j} \int_{\mathbb{R}^{n}} g_{j}(z) d z .
$$

Choose $\varepsilon>0$. We may assume that

$$
\sup _{j} \int_{\mathbb{R}^{n}} g_{j}(z) d z \leq I+\varepsilon
$$

Fix $t>0$ and let

$$
E_{k}=\left\{x \in \mathbb{R}^{n}: \inf _{j \geq k} M g_{j}(x)>t\right\} .
$$

Then $E_{1} \subset E_{2} \subset \ldots$ and

$$
E:=\left\{x \in \mathbb{R}^{n}: M \mathbf{g}(x)>t\right\}=\bigcup_{k} E_{k}
$$


The sets $E_{k}$ are measurable. By the Hardy-Littlewood maximal theorem,

$$
\mathcal{L}^{n}\left(E_{k}\right) \leq \mathcal{L}^{n}\left(\left\{x \in \mathbb{R}^{n}: M g_{k}(x)>t\right\}\right) \leq \frac{C}{t} \int_{\mathbb{R}^{n}} g_{k}(z) d z \leq \frac{C(I+\varepsilon)}{t} .
$$

Letting $E_{k} \nearrow E$ and $\varepsilon \searrow 0$ we conclude

$$
\mathcal{L}^{n}(E) \leq \frac{C I}{t}
$$

Theorem 5. $\mathcal{B} \mathcal{V C}(\Omega) \subset L_{\text {loc }}^{1}(\Omega)$.

Proof. We first show that $u \in \mathcal{B V} \mathcal{C}(\Omega)$ is measurable. It suffices to show that if $u$ is a $B V C$ function in $B_{0}^{\prime}=B\left(x_{0}, 2 r_{0}\right) \subset \Omega$, then $u$ is measurable in $B_{0}=B\left(x_{0}, r_{0} / 2\right)$. Let $\left(g_{j}\right)$ be a $B V$ upper bound for $u$ in $\Omega$ and set $g_{j}=0$ in $\mathbb{R}^{n} \backslash \Omega$.

If $r \in\left(0,3 r_{0} / 2\right), x \in B_{0}$ and $y \in B(x, r)$, then

$|u(y)-u(x)| \leq \liminf _{j} \int_{\gamma_{x, y}} g_{j} d s=\liminf _{j} \int_{0}^{1} g_{j}(x+t(y-x))|y-x| d t$

$$
\leq \liminf _{j} \int_{0}^{1} r g_{j}(x+t(y-x)) d t=: v^{x}(y)
$$

where $\gamma_{x, y}(t)=x+t(y-x), t \in[0,1]$, is the line segment from $x$ to $y$. For a fixed $x$ the function $v^{x}$ is measurable since the functions $g_{j}$ are Borel functions. Now, the Fubini theorem and the Fatou lemma yield

$$
\begin{gathered}
\int_{B(x, r)} v^{x}(y) d y=\int_{B(x, r)}\left(\liminf _{j} \int_{0}^{1} r g_{j}(x+t(y-x)) d t\right) d y \\
\leq \liminf _{j} \int_{B(x, r)}\left(\int_{0}^{1} r g_{j}(x+t(y-x)) d t\right) d y \\
=\liminf _{j} \int_{0}^{1} r t^{-n}\left(\int_{B(x, t r)} g_{j}(z) d z\right) d t \\
\leq \boldsymbol{\alpha}_{n} r^{n+1} \liminf _{j} \int_{0}^{1} M g_{j}(x) d t=\boldsymbol{\alpha}_{n} r^{n+1} M \mathbf{g}(x),
\end{gathered}
$$

where the change of variable from $y$ to $z=x+t(y-x)$ is also used.

To complete the proof let $x_{1}, x_{2} \in B_{0}$ with $r=\left|x_{2}-x_{1}\right|>0$. Write $B=B(a, r / 2)$, where $a=\frac{1}{2}\left(x_{1}+x_{2}\right)$. Then $B \subset B\left(x_{1}, r\right) \cap B\left(x_{2}, r\right) \subset$ $B_{0}^{\prime}$ and for $y \in B$ we have

$$
\left|u\left(x_{2}\right)-u\left(x_{1}\right)\right| \leq\left|u\left(x_{2}\right)-u(y)\right|+\left|u\left(x_{1}\right)-u(y)\right| \leq v^{x_{2}}(y)+v^{x_{1}}(y)
$$

and thus integrating the inequality

$$
\left|u\left(x_{2}\right)-u\left(x_{1}\right)\right| \leq v^{x_{2}}(y)+v^{x_{1}}(y)
$$


over $y \in B$ we obtain from (10)

$$
\boldsymbol{\alpha}_{n}(r / 2)^{n}\left|u\left(x_{2}\right)-u\left(x_{1}\right)\right| \leq \boldsymbol{\alpha}_{n} r^{n+1}\left(M \mathbf{g}\left(x_{1}\right)+M \mathbf{g}\left(x_{2}\right)\right) .
$$

This shows that

$$
\left|u\left(x_{2}\right)-u\left(x_{1}\right)\right| \leq 2^{n}\left|x_{2}-x_{1}\right|\left(M \mathbf{g}\left(x_{1}\right)+M \mathbf{g}\left(x_{2}\right)\right) .
$$

Let $A_{i}=\left\{x \in B_{0}: M \mathbf{g}(x) \leq i\right\}, i=1,2, \ldots$. Then $\mathcal{L}^{n}\left(B_{0} \backslash \cup_{i} A_{i}\right)=0$ as $\mathcal{L}^{n}\left(\left\{x \in B_{0}: M \mathbf{g}(x)=\infty\right\}\right)=0$ by Lemma 4 . In the set $A_{i}$ the function $u$ is Lipschitz and hence measurable and from this it follows that $u$ is measurable in $B_{0}$.

Now it easily follows that $u \in L_{\text {loc }}^{1}(\Omega)$. First note that $|u(x)|<\infty$ for a.e. $x \in \Omega$ by the Fubini theorem, as the family $\Gamma$ of line segments parallel to a coordinate axis on which $u$ fails to be of finite variation has $A M$-modulus zero by Theorem 3 . Then we find $x \in B_{0}$ such that $|u(x)|<\infty$ and $M \mathbf{g}(x)<\infty$. An integration of (9) over $y \in B_{0}$ and (10) show that $u \in L^{1}\left(B_{0}\right)$.

Remark 4. In fact, we can obtain better integrability $\mathcal{B} \mathcal{V C}(\Omega) \subset$ $L_{l o c}^{n /(n-1)}(\Omega)$. This can be obtained following the standard argument by Gagliardo and Nirenberg, or as a consequence of the inclusion $\mathcal{B} \mathcal{V C}(\Omega) \subset B V_{\text {loc }}(\Omega)$ presented later (Theorem 7 ).

\section{4. $B V C$ VERSUS $B V$ FUNCTIONS}

The $M_{1}$-modulus cooperates well with the Sobolev spaces $W^{1,1}$, but not so well with the $B V$ spaces. In this respect the $A M$-modulus is a more efficient tool. Next we show that a precise representative of a $B V$ function in $\Omega$ is a $B V C$ function.

We recall some concepts from the theory of $B V$ functions in $\mathbb{R}^{n}$, see [3] and [8].

A function $u \in L_{\mathrm{loc}}^{1}(\Omega)$ has bounded variation $(B V)$ in $\Omega$ if its distributional gradient $D u$ can be represented as a finite vector-valued signed Radon measure $D u$ in $\Omega$, i.e.

$$
\int_{\Omega} u \operatorname{div} \varphi d x=-\int_{\Omega} \varphi \cdot D u
$$

for every $\varphi \in C_{0}^{1}\left(\Omega, \mathbb{R}^{n}\right)$. Equivalently there are signed Radon measures $D_{i} u, i=1, \ldots, n$, such that

$$
\int_{\Omega} u \partial_{i} \varphi d x=-\int_{\Omega} \varphi D_{i} u
$$

for every $\varphi \in C_{0}^{1}(\Omega)$. We let $|D u|$ and $\left|D_{i} u\right|$ denote the total variation of the measures $D u$ and $D_{i} u, i=1, \ldots, n$, respectively. By the Riesz representation theorem, see e.g. [8], we have

$$
|D u|(\Omega)=\sup \left\{\int_{\Omega} u \operatorname{div} \varphi d x: \varphi \in C_{0}^{1}\left(\Omega, \mathbb{R}^{n}\right),|\varphi| \leq 1\right\}
$$


and for each $i=1, \ldots, n$

$$
\left|D_{i} u\right|(\Omega)=\sup \left\{\int_{\Omega} u \partial_{i} \varphi d x: \varphi \in C_{0}^{1}(\Omega),|\varphi| \leq 1\right\} .
$$

A function $u \in C^{1}(\Omega)$ with $\int_{\Omega}|\nabla u| d x<\infty$ has bounded variation and

$$
|D u|(\Omega)=\int_{\Omega}|\nabla u| d x, \quad\left|D_{i} u\right|(\Omega)=\int_{\Omega}\left|\partial_{i} u\right| d x .
$$

We denote the space of all $B V$ functions in $\Omega$ by $\mathcal{B V}(\Omega)$. This is a Dirichlet type space; we leave the symbol $B V(\Omega)$ for the usual $B V$ space, namely

$$
B V(\Omega)=\mathcal{B V} \mathcal{V}(\Omega) \cap L^{1}(\Omega) .
$$

The space $B V(\Omega)$ is equipped with the $B V$ norm

$$
\|u\|_{B V}=|D u|(\Omega)+\|u\|_{1} .
$$

Similarly, we define

$$
B V C(\Omega)=\mathcal{B} \mathcal{V C}(\Omega) \cap L^{1}(\Omega)
$$

and consider the $B V C$ norm

$$
\|u\|_{B V C}=|D u|_{B V C}(\Omega)+\|u\|_{1} .
$$

Of course, the $B V$ norm does not satisty the axioms of norm on the family of $B V$ functions. For a precise manipulation with the $B V$ space in the framework on normed linear spaces we identify functions which coincide a.e. and mark the underlying linear space

$$
\operatorname{BV}(\Omega):=\left\{[u]_{\mathcal{L}^{n}}: u \in B V(\Omega)\right\},
$$

where

$$
[u]_{\mathcal{L}^{n}}=\{v \in B V(\Omega): v=u \text { a.e. }\}
$$

Concerning the $B V C$ norm, we use a "finer' equivalence relation, so that the underlying linear space is

$$
\operatorname{BVC}(\Omega):=\left\{[u]_{\phi}: u \in B V C(\Omega)\right\},
$$

where

$$
[u]_{\phi}=\{v \in B V C(\Omega): v=u \phi \text {-a.e. }\}
$$

This formalism helps us to explain the diference between the $B V$ and $B V C$ spaces in Remark 5 below.

Let us make the convention that we will still say e.g. " $v$ is a representative of $u \in B V(\Omega)$ " instead of more precise, but clumsy " $v$ is a representative of $[u]_{\mathcal{L}^{n}} \in \mathbf{B V}(\Omega)$ ". if

Let $u \in L_{\text {loc }}^{1}(\Omega)$. A point $z \in \Omega$ is said to be a Lebesgue point for $u$

$$
\lim _{r \rightarrow 0+} f_{B(z, r)}|u(x)-u(z)| d x=0 .
$$

Given $z \in \mathbb{R}^{n}, r>0$ and $\mathbf{n} \in \partial B(0,1)$, we write

$$
B_{\mathbf{n}}(z, r)=\{x \in B(z, r):(x-z) \cdot \mathbf{n}>0\}
$$


and say that $z \in \Omega$ is an $L^{1}$-approximate jump point for $u$ if there exist $a, b \in \mathbb{R}$ and $\mathbf{n} \in \partial B(0,1)$ such that $a \neq b$ and

$$
\lim _{r \rightarrow 0+} f_{B_{\mathbf{n}}(z, r)}|u(x)-b| d x=\lim _{r \rightarrow 0+} f_{B_{-\mathbf{n}}(z, r)}|u(x)-a| d x=0 .
$$

Define the normal vector of $u$ at $z$ by $\mathbf{n}_{u}(z)=(b-a) \mathbf{n}$ where $\mathbf{n}$ is given by the preceding formula; the vector $\mathbf{n}_{u}(z)$ is unique at $L^{1}$-approximate jump points.

We define the $L^{1}$-approximate discontinuity set $S_{u}$ as a set of nonLebesgue points for $u$ and the $L^{1}$-approximate jump set of $J_{u}$ as the set of all $L^{1}$-approximate jump points for $u$; now $J_{u} \subset S_{u}$.

We call $\bar{u}: \Omega \rightarrow \mathbb{R}$ a precise representative of $u$ if

$$
\bar{u}(z)=\lim _{r \rightarrow 0} f_{B(z, r)} u(x) d x
$$

whenever for $z$ this limit exists and is finite. At a jump point $z$ of $u$ we set $\bar{u}(z)=(a+b) / 2$. Now we have $\bar{u}=u$ a.e. in $\Omega$.

If $u$ is a $B V$ function in $\Omega$ and $\bar{u}$ the precise representative of $u$, then by [3, Theorem 3.78] we have $\mathcal{H}^{n-1}\left(S_{u} \backslash J_{u}\right)=0$ and hence by Theorem 3, $A M\left(\Gamma_{S_{u} \backslash J_{u}}\right)=0$ and thus $\bar{u}$ is well defined on $A M$-a.e. path in $\Omega$. The set $S_{u} \backslash J_{u}$ contains all points at which the value of a precise representative remains undetermined. The values of $\bar{u}$ at these points $x$ are immaterial, even can be undefined.

Theorem 6. Let $u$ be a precise representative of a function from $\mathcal{B V}(\Omega)$. Then $u \in \mathcal{B V} \mathcal{C}(\Omega)$ and $u$ has bounded variation on $A M-a . e$. path in $\Omega$. Moreover, there is a sequence of functions $v_{j} \in C^{1}(\Omega)$ such that $v_{j} \rightarrow u$ in $L_{\mathrm{loc}}^{1}(\Omega)$ and $\left|\nabla v_{j}\right|$ is a weak $B V$ upper bound for $u$ with

$$
\begin{aligned}
|D u|(\Omega) & =\lim _{j \rightarrow \infty} \int_{\Omega}\left|\nabla v_{j}\right| d x, \\
\left|D_{i} u\right|(\Omega) & =\lim _{j \rightarrow \infty} \int_{\Omega}\left|\partial_{i} v_{j}\right| d x, \quad i=1, \ldots, n .
\end{aligned}
$$

In particular,

$$
\left|D_{B V C} u\right|(\Omega) \leq|D u|(\Omega)
$$

Proof. As in the proof of [3, Theorem 3.9] or of [8, 5.2.2 Theorem 2] there is a sequence $\left(v_{j}\right)$ of functions $v_{j} \in C^{1}(U)$ such that $v_{j} \rightarrow u$ in $L_{\mathrm{loc}}^{1}(\Omega)$ and (13) holds. The functions $v_{j}$ are constructed by the standard convolution process with a radial mollifier $\eta$, see $[8,4.2 .1$.$] , with$ the aid of a partition of unity which helps to define the approximating function near the boundary. Now at each Lebesgue point $x \in \Omega$ of $u$, i.e. $x \notin S_{u}, v_{j}(x) \rightarrow u(x)$, see [3, Proposition 3.64] or [8, 4.2.1 Theorem 1 ], and also at each jump point $x$ of $u, v_{j}(x) \rightarrow u(x)=(a+b) / 2$, see [3, Proposition 3.69] or [8, 5.9 Corollary 1]. 
Let $E=S_{u} \backslash J_{u}$. Now $A M\left(\Gamma_{E}\right)=0$ and for each path $\gamma$ in $\Omega$ but not in $\Gamma_{E}, v_{i} \circ \gamma(t) \rightarrow u \circ \gamma(t)$ for every $t \in[0, \ell]$ and hence by the lower semicontinuity of the total variation

$$
V(u, \gamma) \leq \liminf _{j} V\left(v_{j}, \gamma\right) \leq \liminf _{j} \int_{\gamma}\left|\nabla v_{j}\right| d s .
$$

Thus the sequence $\left(\left|\nabla v_{j}\right|\right)$ is a weak $B V$ upper bound for $u$ in $\Omega$ and so $u \in \mathcal{B} \mathcal{V C}(\Omega)$.

A converse for Theorem 6 was established in [15, Theorem 5.4]. We will improve the bound obtained there.

Lemma 5. Let $u \in \mathcal{B} \mathcal{V C}(\Omega)$. Let $\left(g_{j}\right)$ a weak $B V$ upper bound for $u$ in $\Omega$. Then $u \in \mathcal{B} \mathcal{V}(\Omega)$ and

$$
|D u(U)| \leq \liminf _{j} \int_{U} g_{j} d x
$$

for each convex open set $U \subset \Omega$ with compact closure in $\Omega$.

Proof. We first fix a convex open set $U \subset \Omega$ with compact closure in $\Omega$ and show that $u \in \mathcal{B} \mathcal{V}(U)$ and, for $i=1, \ldots, n$,

$$
\begin{aligned}
\left|D_{i} u\right|(U) & =\sup \left\{\int_{U} u \partial_{i} \varphi d x: \varphi \in C_{0}^{1}(U),|\varphi| \leq 1\right\} \\
& \leq \liminf _{j} \int_{U} g_{j} d x<\infty .
\end{aligned}
$$

We may assume $i=n$ which simplifies notation. Since $U$ is convex and has compact closure in $\Omega$, for a fixed $y \in \mathbb{R}^{n-1}$, the set $\{t \in \mathbb{R}:(y, t) \in$ $\bar{U}\}$ is a closed interval $L_{y}$ (possibly empty) and from (8) it follows that

$$
V\left(u(y, \cdot), L_{y}\right) \leq \liminf _{j} \int_{L_{y}} g_{j}(y, t) d t
$$

for a.e. $y \in \mathbb{R}^{n-1}$. By Theorem $5, u \in L^{1}(U)$. Let $\varphi \in C_{0}^{1}(U),|\varphi| \leq 1$. Applying the Fubini theorem and the Fatou lemma we obtain from (1) and (16)

$$
\begin{aligned}
\int_{U} u \partial_{n} \varphi d x & =\int_{\mathbb{R}^{n-1}}\left(\int_{L_{y}} u(y, t) \partial_{n} \varphi(y, t) d t\right) d y \\
& \leq \int_{\mathbb{R}^{n-1}} \tilde{V}\left(u(y, \cdot), L_{y}\right) d y \leq \int_{\mathbb{R}^{n-1}} V\left(u(y, \cdot), L_{y}\right) d y \\
& \leq \liminf _{j} \int_{U} g_{j} d x,
\end{aligned}
$$

which verifies (15). To prove (14), pick a unit vector $\mathbf{n}$ such that $|D u(U)|=D u(U) \cdot \mathbf{n}$. We may assume that $\mathbf{n}=\mathbf{e}_{n}$; then

$$
|D u(U)|=D_{n} u(U) \leq \liminf _{j} \int_{U} g_{j} d x
$$

from (15). 
The estimate

$$
\left|D_{i} u\right|(U) \leq \liminf _{j} \int_{U} g_{j} d x, \quad i=1, \ldots, n
$$

from (15) implies

$$
|D u|(B) \leq n \liminf _{j} \int_{B} g_{j} d x
$$

for each ball $B$ with closure in $\Omega$. Using a finite covering with bounded multiplicity of $\Omega$ by such balls and an associated partition of unity we obtain

$$
|D u|(\Omega) \leq C \liminf _{j} \int_{\Omega} g_{j} d x
$$

where $C$ depends only on $n$. Hence $u \in \mathcal{B} \mathcal{V}(\Omega)$.

If $u \in \mathcal{B V}(\Omega)$ is a precisely represented $B V$ function, Theorem 6 shows that $\left|D_{B V C} u\right|(U) \leq|D u|(U)$ for open sets $U \subset \Omega$. In the next theorem we present the converse sharp inequality.

Theorem 7. If $u \in \mathcal{B V} \mathcal{C}(\Omega)$, then $u \in \mathcal{B V}(\Omega)$ and

$$
|D u|(\Omega) \leq\left|D_{B V C} u\right|(\Omega) \text {. }
$$

Proof. Let $\left(g_{j}\right)$ be a $B V$ upper bound for $u$ in $\Omega$. By Lemma $5, u$ is a $B V$ function in $\Omega$. By the Besicovitch differentiation theorem [3, Theorem 2.22], there exists a set $N \subset \Omega$ such that $\Omega \backslash N$ is contained in the support of $|D u|$,

$$
|D u|(\Omega \cap N)=0
$$

and for each $y \in \Omega \backslash N$ there exists

$$
\lim _{r \rightarrow 0} \frac{D u(B(y, r))}{|D u|(B(y, r)} \in \partial B(0,1) .
$$

Given $\varepsilon>0$, with each $y \in \Omega$ we associate the family $R(y)$ of all radii $r>0$ with the properties $\bar{B}(y, r) \subset \Omega$,

$$
|D u|(B(y, r)) \leq(1+\varepsilon)|D u(B(y, r))|
$$

and

$$
|D u|(\partial B(y, r))=0 .
$$

This defines a fine centered covering of $\Omega \backslash N$. By the Vitali-Besicovitch covering theorem [3, Theorem 2.19], there exists a countable (possibly finite) pairwise disjoint collection $\left\{B\left(y_{i}, r_{i}\right)\right\}$ of balls such that $y_{i} \in$ $\Omega \backslash N, r_{i} \in R\left(y_{i}\right)$ and

$$
|D u|\left((\Omega \backslash N) \backslash \bigcup_{i} \bar{B}\left(y_{i}, r_{i}\right)\right)=0 .
$$


However, since $|D u|(N)=0$ and in view of (22), we can improve to

$$
|D u|\left(\Omega \backslash \bigcup_{i} B\left(y_{i}, r_{i}\right)\right)=0
$$

Now Lemma 5 and (21) yield

$$
\begin{aligned}
& |D u|(\Omega) \leq \sum_{i}|D u|\left(B\left(y_{i}, r_{i}\right)\right) \leq(1+\varepsilon) \sum_{i}\left|D u\left(B\left(y_{i}, r_{i}\right)\right)\right| \\
\leq & (1+\varepsilon) \sum_{i} \liminf _{j} \int_{\left.B\left(y_{i}, r_{i}\right)\right)} g_{j} d x \leq(1+\varepsilon) \liminf _{j} \int_{\Omega} g_{j} d x .
\end{aligned}
$$

Since $\left(g_{j}\right)$ was arbitrary, letting $\varepsilon \rightarrow 0$ we obtain the desired inequality.

The above results lead to the following characterization of $B V$ functions.

Theorem 8. Let $u$ be a function on $\Omega$. Then $u$ is a $B V$ function in $\Omega$ if and only if there exists a $B V C$ function $v$ on $\Omega$ such that $u=v$ a.e.

Proof. This is immediate from Theorem 6 and Theorem 7 .

Remark 5. We have proved inclusions

$$
\{u \in B V(\Omega): u \text { is precisely represented }\} \subset B V C(\Omega) \subset B V(\Omega) .
$$

These inclusions are both strict: The characteristic function of a singleton is in $B V C(\Omega)$ but it is not precisely represented. The characteristic function of the set of all $x \in \Omega$ which have at least one rational coordinate is in $B V(\Omega)$ but not in $B V C(\Omega)$. Let us associate a precise representative $\bar{u}$ with any $u \in B V(\Omega)$. Consider the induced mappings

$$
\begin{gathered}
\epsilon:[u]_{\mathcal{L}^{n}} \mapsto[\bar{u}]_{\phi}: \operatorname{BV}(\Omega) \rightarrow \operatorname{BVC}(\Omega), \\
\varkappa:[u]_{\phi} \mapsto[u]_{\mathcal{L}^{n}}: \operatorname{BVC}(\Omega) \rightarrow \operatorname{BV}(\Omega) .
\end{gathered}
$$

If $v_{1}, v_{2}$ are precise representatives of $u \in B V(\Omega)$, then $v_{1}=v_{2} \mathcal{H}^{n-1}$ a.e. and the more $v_{1}=v_{2} \phi$-a.e., so that $\epsilon$ is well defined. Both $\epsilon$ and $\varkappa$ are linear. We will show that $\epsilon$ is injective, but not surjective and $\varkappa$ is surjective, but not injective. Therefore we cannot identify the spaces $\mathbf{B V C}(\Omega)$ and $\mathbf{B V}(\Omega)$. Indeed, the injectivity of $\epsilon$ is obvious. For any $\mathbf{u} \in \mathbf{B V}(\Omega)$ we have $\mathbf{u}=\varkappa(\epsilon(\mathbf{u}))$, so that $\varkappa$ is surjective. Now, consider the characteristic function $u$ of the singleton $\{0\}$ in $(-1,1)$. Then $\phi(\{0\})=1$, hence $[u]_{\phi} \neq[0]_{\phi}$, but

$$
\varkappa\left([u]_{\phi}\right)=[0]_{\mathcal{L}^{n}}=\varkappa\left([0]_{\phi}\right) .
$$

Therefore $\varkappa$ is not injective. The same $u$ disproves surjectivity of $\epsilon$, as $[u]_{\phi}$ does not belong to its range. Note that $\left|D_{B V C} u\right|((-1,1))=2$.

The representatives $u$ of $u \in B V(\Omega)$ can be classified as non- $B V C$ representatives, "bad" $B V C$ representatives and "good" $B V C$ representatives. Here, we mean that $u$ is a good representative if $[u]_{\phi}=$ 
$\epsilon\left([u]_{\mathcal{L}^{n}}\right)$. If $u$ is a good representative, then $|D u|(\Omega)=\left|D_{B V C} u\right|(\Omega)$ (but not conversely, consider the characteristic function of $[0,1)$ in $(-1,1))$.

\section{5. $A M-$ MOdulus AND $(n-1)$-RECTIFIABle SETS}

A set $A \subset \mathbb{R}^{n}$ is called countably $\mathcal{H}^{n-1}$-rectifiable, or (following [16]) simply $(n-1)$-rectifiable, if there are Lipschitz maps $h_{i}: \mathbb{R}^{n-1} \rightarrow \mathbb{R}^{n}$, $i=1,2, \ldots$, such that $\mathcal{H}^{n-1}\left(A \backslash \cup_{i=1}^{\infty} h_{i}\left(\mathbb{R}^{n-1}\right)\right)=0$.

For the theory of rectifiable sets see [9], [16] and [3].

We first state the lower estimate as a separate lemma.

Lemma 6. Suppose that $A \subset \mathbb{R}^{n}$ is $(n-1)$-rectifiable. If $E \subset A$, then

$$
\mathcal{H}^{n-1}(E) \leq A M\left(\Gamma_{E}^{c}\right)
$$

Proof. Assume first that $E$ is $\mathcal{H}^{n-1}$-measurable. The result of $[3$, Proposition 2.66] states that (due to $(n-1)$-rectifiability)

$$
\begin{aligned}
\mathcal{H}^{n-1}(E)=\sup \{ & \sum_{i=1}^{\infty} \mathcal{H}^{n-1}\left(\Pi_{V_{i}}\left(K_{i}\right)\right): V_{i} \in G(n, n-1), \\
& \left.K_{i} \subset E \text { compact and pairwise disjoint }\right\} .
\end{aligned}
$$

If $K_{i}$ and $V_{i}$ are as above, by (4) we have

$$
\sum_{i=1}^{\infty} \mathcal{H}^{n-1}\left(\Pi_{V_{i}}\left(K_{i}\right)\right) \leq \sum_{i=1}^{\infty} \phi\left(K_{i}\right) \leq \phi(E) .
$$

Hence $\mathcal{H}^{n-1}(E) \leq \phi(E)$ and in the general case we use Theorem 2 to find a $\phi$-measurable set $F \supset E$ such that $\phi(F)=\phi(E)$ and obtain

$$
\mathcal{H}^{n-1}(E) \leq \mathcal{H}^{n-1}(F) \leq \phi(F)=\phi(E) .
$$

Comparing the previous lemma with Theorem 3 we already know that $\mathcal{H}^{n-1}$ and $\phi$ are comparable on $(n-1)$-rectifiable sets. However, we can profit from rectifiability also in the sharp constant of the upper estimate and prove that $\mathcal{H}^{n-1}$ and $\phi$ in fact agree on $(n-1)$-rectifiable sets.

We start with a simple estimate of Minkowski content. If $E \subset \mathbb{R}^{n}$ is an arbitrary set, we define

$$
E_{r}=\left\{x \in \mathbb{R}^{n}: \operatorname{dist}(x, E)<r\right\}
$$

and

$$
\underline{\mathcal{M}}^{n-1}(E)=\liminf _{r \rightarrow 0+} \frac{\mathcal{L}^{n}\left(E_{r}\right)}{2 r} .
$$

This is the lower Minkowski content of E.

Lemma 7. $\phi(E) \leq \underline{\mathcal{M}}^{n-1}(E)$ for every set $E \subset \mathbb{R}^{n}$ 
Proof. We find a sequence $\left(r_{i}\right), r_{i} \searrow 0$, such that

$$
\frac{\mathcal{L}^{n}\left(E_{r_{i}}\right)}{2 r_{i}} \rightarrow \underline{\mathcal{M}}^{n-1}(E)
$$

and set $\rho_{i}=\chi_{E_{r_{i}}} /\left(2 r_{i}\right)$. Let $\gamma \in \Gamma_{E}^{c}$. Then there exists $i_{0} \in \mathbb{N}$ and $t \in(0, \ell)$ such that $\gamma(t) \in E$ and $\left[t-r_{i}, t+r_{i}\right] \subset[0, \ell]$ for each $i \geq i_{0}$. Since (by our convention) $\gamma$ is parametrized by its arclength, we have $\gamma\left(\left[t-r_{i}, t+r_{i}\right]\right) \subset E_{r_{i}}$ and thus

$$
\int_{\gamma} \rho_{i} \geq 1, \quad i \geq i_{0}
$$

It follows that $\left(\rho_{i}\right)$ is admissible for $\Gamma_{E}^{c}$ and thus

$$
\phi(E) \leq \liminf _{j} \int_{\mathbb{R}^{n}} \rho_{i} d x=\liminf _{j} \frac{\mathcal{L}^{n}\left(E_{r_{i}}\right)}{2 r_{i}}=\underline{\mathcal{M}}^{n-1}(E) .
$$

Theorem 9. If $A \subset \mathbb{R}^{n}$ is $(n-1)$-rectifiable and $E \subset A$, then

$$
\mathcal{H}^{n-1}(E)=A M\left(\Gamma_{E}^{c}\right) \text {. }
$$

Proof. By Theorem 3 it suffices to show

$$
A M\left(\Gamma_{E}^{c}\right) \leq \mathcal{H}^{n-1}(E)
$$

and we may assume that $\mathcal{H}^{n-1}(E)<\infty$. Also assume first that $E$ is $\mathcal{H}^{n-1}$-measurable. Then as a straightforward consequence of the definition, there exist compact sets $K_{j} \subset \mathbb{R}^{n-1}$ and Lipschitz maps $f_{j}: K_{j} \rightarrow \mathbb{R}^{n}$ such that $f_{j}\left(K_{j}\right)$ are pairwise disjoint subsets of $E$ and

$$
\mathcal{H}^{n-1}\left(E \backslash \bigcup_{j} f_{j}\left(K_{j}\right)\right)=0
$$

Theorem $[9,3.2 .39]$ states that $\underline{\mathcal{M}}^{n-1}(F)=\mathcal{H}^{n-1}(F)$ for each Lipschitz image of a compact subset of $\mathbb{R}^{n-1}$. Hence by Lemma 7 ,

$$
\begin{aligned}
\phi(E) & =\sum_{j} \phi\left(f_{j}\left(K_{j}\right)\right) \leq \sum_{j} \underline{\mathcal{M}}^{n-1}\left(f_{j}\left(K_{j}\right)\right) \\
& =\sum_{j} \mathcal{H}^{n-1}\left(f_{j}\left(K_{j}\right)\right) \leq \mathcal{H}^{n-1}(E) .
\end{aligned}
$$

Here we have used that $\phi(N)=0$ for each $N \subset \mathbb{R}^{n}$ with $\mathcal{H}^{n-1}(N)=0$, this follows from Theorem 3.

Now we may remove the additional assumption of $\mathcal{H}^{n-1}$-measurability. Indeed, since $\mathcal{H}^{n-1}$ is a Borel regular measure, for an arbitrary set $E \subset A$ there exists a Borel set $F \supset E$ such that $\mathcal{H}^{n-1}(F)=\mathcal{H}^{n-1}(E)$ and we can use the estimate above for $F \cap A$. 


\section{Perimeter and $A M-$ modulus}

We first recall the basic concepts on sets of finite perimeter, see [3] and [8].

Let $E \subset \mathbb{R}^{n}$ be a Lebesgue measurable set. The measure theoretic boundary $\partial_{*} E$ of $E$ consists of points $x \in \mathbb{R}^{n}$ such that $\Theta^{n}(x, E)>0$ and $\Theta^{n}\left(x, \mathbb{R}^{n} \backslash E\right)>0$ where

$$
\Theta^{n}(x, A)=\limsup _{r \rightarrow 0} \frac{\mathcal{L}^{n}(B(x, r) \cap A)}{\mathcal{L}^{n}(B(x, r))}
$$

is the upper Lebesgue density of $A$ at $x$. The measure theoretic interior int $_{*} E$ of $E$ and the measure theoretic exterior ext $_{*} E$ are the sets of points $x \in \mathbb{R}^{n}$ where $\Theta^{n}\left(x, \mathbb{R}^{n} \backslash E\right)=0$ and $\Theta^{n}(x, E)=0$, respectively. The sets $\partial_{*} E$, int ${ }_{*} E$ and ext $_{*} E$ are Borel sets.

A set $E \subset \mathbb{R}^{n}$ has finite perimeter if $\chi_{E} \in B V\left(\mathbb{R}^{n}\right)$, i.e. $E$ has finite Lebesgue measure and $P(E)=\left|D \chi_{E}\right|\left(\mathbb{R}^{n}\right)<\infty$.

It is also possible to consider sets $E$ which have finite perimeter in an open set $\Omega \subset \mathbb{R}^{n}$, i.e. $\chi_{E}$ is a $B V$ function in $\Omega$, but, for simplicity, we only consider $\Omega=\mathbb{R}^{n}$.

If $E$ has finite perimeter we let $\partial^{*} E$ denote the reduced boundary of $E$, which consist of all points $x$ from the support of $\left|D \chi_{E}\right|$ for which there exists a unit vector $\mathbf{n}(x)$ such that

$$
\lim _{r \rightarrow 0} \frac{D \chi_{E}(B(x, r))}{\left|D \chi_{E}(B(x, r))\right|}=\mathbf{n}(x),
$$

see [3, Definition 3.54] $[8,5.7 .1]$. Then we have

$$
\left|D \chi_{E}\right|(A)=\mathcal{H}^{n-1}\left(\partial^{*} E \cap A\right)=\mathcal{H}^{n-1}\left(\partial_{*} E \cap A\right)
$$

for each Borel set $A \subset \mathbb{R}^{n}$, see [3, Theorem 3.59 and Theorem 3.61] or [8, 5.7.3 and 5.8]. From Theorems 6 and 7 we obtain

Corollary 1. If $E$ has finite perimeter, then the precise representative $\bar{\chi}_{E}$ of $\chi_{E}$ belongs to $B V C\left(\mathbb{R}^{n}\right)$ and, conversely, if $\chi_{E} \in B V C\left(\mathbb{R}^{n}\right)$, then $E$ has finite perimeter.

Next we use the results from the previous sections to express $\left|D \chi_{E}\right|(A)$ for sets $A \subset \mathbb{R}^{n}$ in terms of the $A M$-modulus.

Theorem 10. Suppose that $E$ has finite perimeter and $A \subset \mathbb{R}^{n}$ a Borel set. Then

$$
\left|D \chi_{E}\right|(A)=A M\left(\Gamma_{A \cap \partial_{*} E}^{c}\right)
$$

and, in particular, $P(E)=A M\left(\Gamma_{\partial_{*} E}^{c}\right)$.

Proof. By the structure theorem for sets of finite perimeter, see $[3$, Theorem 3.59] or [8, 5.7.3 Theorem 2], the reduced boundary $\partial^{*} E$ is $(n-1)$-rectifiable and $\left|D \chi_{E}\right|(A)=\mathcal{H}^{n-1}\left(A \cap \partial^{*} E\right)$. Since $\partial^{*} E \subset \partial_{*} E$ and $\mathcal{H}^{n-1}\left(\partial_{*} E \backslash \partial^{*} E\right)=0$, by [3, Theorem 3.61] or [8, 5.8 Lemma 1], 
$\left|D \chi_{E}\right|(A)=\mathcal{H}^{n-1}\left(A \cap \partial_{*} E\right)$. Now the proof follows from Theorem 9 .

Next we characterize the sets $E$ of finite perimeter in terms of the path families $\Gamma_{\partial_{*} E}^{c}$ and $\Gamma_{\text {cross }}(E)$, where the latter family consists of all paths in $\mathbb{R}^{n}$ connecting int ${ }_{*} E$ to ext $_{*} E$, i.e. each path in $\Gamma_{\text {cross }}(E)$ meets both $\operatorname{int}_{*} E$ and ext $_{*} E$.

Let $E \subset \mathbb{R}^{n}$ be a set of finite measure. We start by showing that the condition $A M\left(\Gamma_{\text {cross }}(E)\right)<\infty$ implies that $E$ has finite perimeter. Then we proceed to prove a sharp result in Theorem 11.

Lemma 8. If $A M\left(\Gamma_{\text {cross }}(E)\right)<\infty$, then $E$ has finite perimeter. Moreover, if $\left(\rho_{j}\right)$ is an admissible sequence for $\Gamma_{\text {cross }}(E)$, then for each ball $B(z, r)$ and every $i \in\{1, \ldots, n\}$

$$
\left|D_{i} \chi_{E}\right|\left(B(z, r) \leq \liminf _{j} \int_{B(z, r)} \rho_{j} d x\right.
$$

and

$$
\left|D \chi_{E}(B(z, r))\right| \leq \liminf _{j} \int_{B(z, r)} \rho_{j} d x
$$

Proof. Assume that $\chi_{E}$ is precisely represented. Fix a ball $B=B(z, r)$ and $i \in\{1, \ldots, n\}$ and let $\Pi_{i}$ denote the orthogonal projection of $\mathbb{R}^{n}$ onto the $i^{\text {th }}$-coordinate plane. Given $y \in \Pi_{i}(B)$, we let $\gamma_{y}:[0, \ell] \rightarrow \bar{B}$ denote the path which parametrizes the line segment $L_{y}$ parallel to the $x_{i}$-axis, joins the opposite faces of $\partial B$ and whose continuation passes through $y$. Since the set $\partial_{*} E$ has Lebesgue measure zero, the Fubini theorem yields that for a.e. $y \in \Pi_{i}(B)$, the set $L_{y} \cap \partial_{*} E$ has one-dimensional measure zero. Therefore, for any $\lambda<\tilde{V}\left(\chi_{E}, \gamma_{y}\right)$ there exists a partition $0<t_{0}<t_{1}<\cdots<t_{m}<\ell$ such that $m \geq \lambda$, all the points $\gamma_{y}\left(t_{\alpha}\right)$ belong to $\operatorname{int}_{*} E \cup \operatorname{ext}_{*} E$, and

$$
\left|\chi_{E}\left(\gamma_{y}\left(t_{\alpha}\right)\right)-\chi_{E}\left(\gamma_{y}\left(t_{\alpha-1}\right)\right)\right| \geq 1, \quad \alpha=1, \ldots, m .
$$

Set

$$
\gamma_{y, \alpha}(t)=\gamma_{y}\left(t-t_{\alpha-1}\right), \quad t \in\left[0, t_{\alpha}-t_{\alpha-1}\right], \quad \alpha=1, \ldots, m .
$$

Then each $\gamma_{y, \alpha}$ belongs to $\Gamma_{\text {cross }}(E)$ and thus

$$
\begin{aligned}
& 1 \leq \liminf _{j} \int_{\gamma_{y, \alpha}} \rho_{j} d s, \quad \alpha=1, \ldots, m, \\
& \lambda \leq \sum_{\alpha=1}^{m} \liminf _{j} \int_{\gamma_{y, \alpha}} \rho_{j} d s \leq \liminf _{j} \int_{\gamma_{y}} \rho_{j} d s .
\end{aligned}
$$

Then letting $\lambda \rightarrow \tilde{V}\left(\chi_{E}, \gamma_{y}\right)$ we obtain

$$
\tilde{V}\left(\chi_{E}, \gamma_{y}\right) \leq \liminf _{j} \int_{\gamma_{y}} \rho_{j} d s
$$


and integrating with respect to $y$ and using the Fatou lemma and the Fubini theorem we conclude that

$$
\int_{\Pi_{i}(B)} \tilde{V}\left(\chi_{E}, \gamma_{y}\right) d y \leq \int_{\Pi_{i}(B)}\left(\liminf _{j} \int_{\gamma_{y}} \rho_{j} d s\right) d y \leq \liminf _{j} \int_{B} \rho_{j} d x .
$$

Now from (12), (1) and the Fubini theorem we obtain (28). Note that $y \mapsto \tilde{V}\left(\chi_{E}, \gamma_{y}\right)$ is $\mathcal{L}^{n-1}$-measurable, see [8, 5.10.2 Lemma 1].

Since (28) is true in every ball, we have

$$
\left|D_{i} \chi_{E}\right|\left(\mathbb{R}^{n}\right) \leq \liminf _{j} \int_{\mathbb{R}^{n}} \rho_{j} d x
$$

for every $i \in\{1, \ldots, n\}$ and this implies that $P(E)=\left|D \chi_{E}\right|\left(\mathbb{R}^{n}\right)<\infty$.

To prove (29) note that there exists a unit vector $\mathbf{n}$ such that

$$
\left|D \chi_{E}(B)\right|=D \chi_{E}(B) \cdot \mathbf{n} \text {. }
$$

We may assume that $\mathbf{n}=\mathbf{e}_{n}$ and then $D \chi_{E}(B) \cdot \mathbf{n}=D_{n} \chi_{E}(B)$. Since $\left|D_{n} \chi_{E}(B)\right| \leq\left|D_{n} \chi_{E}\right|(B)$ the result follows from (29).

Theorem 11. If $E \subset \mathbb{R}^{n}$ is a set of finite (Lebesgue) measure, then

$$
A M\left(\Gamma_{\partial_{*} E}^{c}\right)=P(E)=A M\left(\Gamma_{\text {cross }}(E)\right) .
$$

Proof. If $P(E)<\infty$, then Theorem 10 gives $P(E)=A M\left(\Gamma_{\partial_{*} E}^{c}\right)$. Suppose that $A M\left(\Gamma_{\partial_{*} E}^{c}\right)<\infty$. By Theorem $4, \mathcal{G}_{n-1}\left(\partial_{*} E\right)<\infty$ and since the $(n-1)$-dimensional integral geometric measure (with exponent 1 ) $\mathcal{I}_{1}^{n-1}$ satisfies $\mathcal{I}_{1}^{n-1} \leq \mathcal{G}_{n-1}$, see $[9,2.10 .5-6], \mathcal{I}_{1}^{n-1}\left(\partial_{*} E\right)<\infty$. Now, by $[9,4.5 .11]$ it follows that $E$ has finite perimeter (for a more elementary proof of this deep result see [7, Theorem 4.9]). Then Theorem 10 gives $A M\left(\Gamma_{\partial_{*} E}^{c}\right)=P(E)$.

It remains to consider the second equality in (30). We first show that

$$
P(E) \leq A M\left(\Gamma_{\text {cross }}(E)\right) .
$$

We may assume that $A M\left(\Gamma_{\text {cross }}(E)\right)<\infty$. Then Lemma 8 yields that $\chi_{E} \in B V\left(\mathbb{R}^{n}\right)$ and we replace $\chi_{E}$ by its precise representative. Let $\left(\rho_{j}\right)$ be an admissible sequence for $\Gamma_{\text {cross }}(E)$. Now we can proceed as in the proof of Theorem 7. Given $\varepsilon>0$, using the Vitali-Besicovitch covering theorem [3, Theorem 2.19], the definition of reduced boundary, (26) and (29) we find a countable (possibly finite) pairwise disjoint collection $\left\{B\left(y_{i}, r_{i}\right)\right\}$ of balls such that

$$
\begin{gathered}
\left|D \chi_{E}\right|\left(\partial^{*} E \backslash \bigcup_{i} B\left(y_{i}, r_{i}\right)\right)=0, \\
\left|D \chi_{E}\right|\left(B\left(y_{i}, r_{i}\right)\right) \leq(1+\varepsilon)\left|D \chi_{E}\left(B\left(y_{i}, r_{i}\right)\right)\right|,
\end{gathered}
$$

and

$$
\left|D \chi_{E}\left(B\left(y_{i}, r_{i}\right)\right)\right| \leq \liminf _{j} \int_{B\left(y_{i}, r_{i}\right)} \rho_{j} d x .
$$


Summing over $i$ we obtain

$$
P(E) \leq(1+\varepsilon) \liminf _{j} \int_{\mathbb{R}^{n}} \rho_{j} d x
$$

and since this holds for every $\varepsilon>0$ and every admissible sequence $\left(\rho_{j}\right)$, (31) follows.

To prove the reverse inequality to (31) we may assume that $P(E)<$ $\infty$. Now we can use the fact that $\chi_{E}$ is a $B V$ function. By the approximation theorem for $B V$ functions ([3, Theorem 3.9] or [8, 5.2.2 Theorem 2]) a sequence of BV functions $f_{i} \in C^{1}\left(\mathbb{R}^{n}, \mathbb{R}\right)$ such that

$$
\int_{\mathbb{R}^{n}}\left|\nabla f_{i}\right| d x \rightarrow P(E)
$$

and $f_{i}(x) \rightarrow \chi_{E}(x)$ at every density point $x$ of $E$ and at every density point $x$ of $\mathbb{R}^{n} \backslash E$ as $i \rightarrow \infty$. Now the sequence $\left(\left|\nabla f_{i}\right|\right)$ is an $A M-$ admissible sequence for $\Gamma_{\text {cross }}(E)$ because for $\gamma \in \Gamma_{\text {cross }}(E)$ we find $t_{1}$ and $t_{2}$ such that $\gamma\left(t_{1}\right) \in \operatorname{ext}_{*} E$ and $\gamma\left(t_{2}\right) \in$ int $_{*} E$ and thus

$$
1=\lim _{i}\left|f_{i}\left(\gamma\left(t_{2}\right)\right)-f_{i}\left(\gamma\left(t_{1}\right)\right)\right| \leq \liminf _{i} \int_{\gamma}\left|\nabla f_{i}\right| d s
$$

and we obtain

$$
A M\left(\Gamma_{\text {cross }}(E)\right) \leq \liminf _{i} \int_{\mathbb{R}^{n}}\left|\nabla f_{i}\right| d x=P(E) .
$$

\section{ReFERENCES}

[1] L. Ambrosio and S. Di Marino, Equivalent definitions of BV space and of total variation on metric measure spaces. J. Funct. Anal. 266 (2014), no. 7, 4150-4188.

[2] L. Ambrosio, S. Di Marino and G. Savaré, On the duality between p-modulus and probability measures. J. Eur. Math. Soc. (JEMS) 17 (2015), no. 8, 18171853.

[3] L. Ambrosio, N. Fusco and D. Pallara, Functions of bounded variation and free discontinuity problems. Oxford Mathematical Monographs, Calendron Press, New York 2000.

[4] L. Ambrosio, N. Gigli and G. Savaré, Density of Lipschitz functions and equivalence of weak gradients in metric measure spaces. Rev. Mat. Iberoam. 29 (2013), no. 3, 969-996.

[5] A. Björn, J. Björn, Nonlinear Potential Theory on Metric Spaces. EMS Tracts in Mathematics, 17. European Mathematical Society (EMS), Zurich, 2011.

[6] A. Björn, J. Björn and J. Malý, Quasiopen and p-Path Open Sets, and Characterizations of Quasicontinuity. Potential Anal. 46 (2017), no. 1, 181199.

[7] M. Chlebík, Going beyond variation of sets. arXiv:1611.05931v1 [math.MG] 17 Nov 2016.

[8] L. C. Evans and R. Gariepy, Measure Theory and Fine Properties of Functions. Studies in Advanced Mathematics, CRC Press, Boca Raton, 1992.

[9] H. Federer, Geometric Measure Theory. Springer-Verlag, 1969. 
[10] B. Fuglede, Extremal length and functional completion. Acta Math. 98 (1957), 171-219.

[11] V. Honzlová Exnerová, J. Malý and O. Martio, Modulus in Banach Function Spaces. Ark. Mat. 55.1 (2017), 105-130.

[12] E. Järvenpää, M. Järvenpää, K. Rogovin, S. Rogovin and N. Shanmugalingam, Measurability of equivalence classes and MECp-property in metric spaces. Rev. Mat. Iberoam. 23 (2007), no. 3, 811-830.

[13] A. S. Kechris, Classical Descriptive Set Theory. Graduate Texts in Mathematics, Springer, 1995.

[14] O. Martio, Functions of bounded variation and curves in metric measure spaces. Adv. Calc. Var., Vol. 9, Issue 4 (2016), 305-322.

[15] O. Martio, The space of functions of bounded variation on curves in metric measure spaces. Conform. Geom. Dyn. 20 (2016), 81-96.

[16] P. Mattila, Geometry of Sets and Measures in Euclidean Spaces: Fractals and Rectifiability. Cambridge University Press, 1995.

[17] W. Sierpiński, Sur un problème concernant les ensembles mesurables superficiellement, Fundamenta Mathematicae, Vol. 1, Issue 1 (1920), 112-115.

Department of Mathematical Analysis, Faculty of Mathematics and Physics, Charles University in Prague, Sokolovská 83, Prague 8, 18675 Czech Republic

E-mail address: venda.ex@gmail.com

Department of Mathematical Analysis, Faculty of Mathematics and Physics, Charles University in Prague, Sokolovská 83, Prague 8, 18675 Czech Republic

E-mail address: maly@karlin.mff.cuni.cz

Department of Mathematics and Statistics, FI-00014 University of HELSINKI, FinLAND

E-mail address: olli.martio@helsinki.fi 